\title{
The Mass Calculation of Solitary Wave Solution of the One-Dimensional Burgers Equation
}

\author{
T. B. Prayitno*) \\ *) Physics Department, Universitas Negeri Jakarta, Jl. Pemuda Rawamangun no. 10, Jakarta, 13220 \\ E-mail : trunk_002@yahoo.com
}

\begin{abstract}
We have calculated the mass of the solitary wave solution of the one-dimensional Burgers equation by integrating the Hamiltonian density of its equation based on the formulation of the classical field theory. To use this method, we first construct the Lagrangian density in order to obtain the Hamiltonian density by initially introducing the ansatz function of the appropriate field. In this paper, we have obtained that the mass of the solitary of the one-dimensional of Burgers equation is literally divergent.
\end{abstract}

\section{Keywords: Solitary wave, Burgers equation.}

\section{Introduction}

The phenomenons of the solitary wave solutions have been well-known in physics since a few centuries ago. The special solution itself can only be obtained by solving the nonlinear wave equation by imposing the boundary conditions either analytic or numeric. It was J. Scott Russel who initially observed the phenomenon in 1834 when he viewed the water wave in a narrow canal $[1,2]$. The wave traveled with the constant form and velocity without distortion. The wave brought the accumulation of water that can be viewed as the energy of the water wave. In a few years later, Korteweg and de Vries proposed their first analytical solution in order to explain the above phenomenon. The proposed equation achieving the above solution physically contains the nonlinear and dispersive terms but neglects the dissipation [2]. In other words, the solitary wave can be defined as a stable wave solution propagating without distortion since the nonlinear and dispersive aspects cancel each other.

Later, the nonlinear wave equations that creating the solitary wave solutions have been used as a mathematical tool to deeply understand the characteristics of waves that propagating in the medium. One of the nonlinear wave equations which has been studied in physics is the Burgers equation. The Burgers equation itself was initially introduced by J. M Burgers to discuss the phenomenon of nonlinear diffusion [3]. Although the solutions of the Burgers equation can be obtained by imposing the boundary conditions, this time we are only interested in the solitary wave solution. In fact, the solitary wave solution of the onedimensional Burgers equation can be analytically obtained by initially applying the Cole-Hopf transformation in order to reduce it into linear differential equation [4]. It is also proven that the solution can be considered as a kink solution. Interested readers can more deeply study about Burgers equation for example in Ref. [5-7]

According to Classical Field Theory (CST), the mass of the solitary wave solution can be calculated analytically by integrating the Hamiltonian density over the space [8]. Interestingly, the above definition brings us to the dualism particle and wave as in quantum mechanics. It can be stated that the solitary wave solution can be considered as a particle. Some paper have been used the above characteristics in order to study to model of hadrons [9-11]. This time, we concern to calculate the mass of the one-dimensional Burgers equation by following the method written by Wospakrik [5]. We organize the paper as follows: we construct the Lagrangian density and Hamiltonian density for the onedimensional Burgers equation in Sec. II. In Sec. III, we review some characteristics of the onedimensional Burgers equation, including its solitary wave solution. 


\section{Methods}

In this section, we review and construct some formulation that can be applied in the next section. In our previous paper [12], it has been obtained both the Lagrangian density and Hamiltonian density in the form of $(1+3)$ spacetime dimensions derived by minimizing the action, imposing the appropriate boundary conditions, and applying the transformations both for the field and space-time coordinates. Firstly, we rewrite the previous results and reduce them into $(1+1)$ space-time dimensions. We initially consider the ansatz Lagrangian which can be written as

$$
L=L\left(\varphi, \partial_{\mu} \varphi, \partial^{\mu} \partial_{\mu} \varphi, \partial^{\alpha} \partial^{\mu} \partial_{\mu} \varphi, x^{\mu}\right) \text {. }
$$

Then, the variation of the action function can be written as

$$
\delta S=\int_{R}\left(\delta L+L \partial_{\mu} \delta x^{\mu}\right) d^{4} x,
$$

with $R$ is the $(1+3)$ space-time region that the Lagrangian density is defined and the variation of the Lagrangian density has the form

$$
\begin{aligned}
\delta L= & \frac{\partial L}{\partial \varphi} \delta \varphi+\frac{\partial L}{\partial\left(\partial_{\mu} \varphi\right)} \delta\left(\partial_{\mu} \varphi\right) \\
& +\frac{\partial L}{\partial\left(\partial^{\alpha} \partial_{\alpha} \varphi\right)} \delta\left(\partial^{\mu} \partial_{\mu} \varphi\right)+\frac{\partial L}{\partial x^{\mu}} \delta\left(\partial x^{\mu}\right) \\
& +\frac{\partial L}{\partial\left(\partial^{\alpha} \partial^{\beta} \partial_{\beta} \varphi\right)} \delta\left(\partial^{\alpha} \partial^{\beta} \partial_{\mu} \varphi\right)
\end{aligned}
$$

Here we choose that $\mu$ runs 0 to 3 also write $\eta_{\mu v}=(+----)$, so $x^{0}=x_{0}=t, x^{1}=-x_{1}=x$, $x^{2}=-x_{2}=y, x^{3}=-x_{3}=z$.

By applying the Gauss theorem in Eq. (2) and imposing the boundary conditions on the surface of $R$

$$
\delta x^{\mu}=0 \text { and } \delta \varphi=0,
$$

we obtain the Euler-Lagrange for the Lagrangian density as

$$
\begin{gathered}
\frac{\partial L}{\partial \varphi}-\partial_{\mu}\left(\frac{\partial L}{\partial\left(\partial_{\mu} \varphi\right)}\right)+\partial^{\mu} \partial_{\mu}\left(\frac{\partial L}{\partial\left(\partial^{\alpha} \partial_{\alpha} \varphi\right)}\right) \\
-\partial^{\mu} \partial_{\mu} \partial^{\alpha}\left(\frac{\partial L}{\partial\left(\partial^{\alpha} \partial_{\alpha} \partial_{\beta} \varphi\right)}\right)=0
\end{gathered}
$$

It must be noted that we have used the transformations for space-time coordinates and field as follows

$$
\begin{gathered}
x^{\prime \alpha}=x^{\alpha}+\delta x^{\alpha}, \\
\varphi\left(x^{\prime \alpha}\right)=\varphi\left(x^{\alpha}\right)+\delta \varphi\left(x^{\alpha}\right) .
\end{gathered}
$$

In field theory, the Hamiltonian density is associated by the zero component of the energy-momentum tensor [13]. Therefore, by extending the transformation of field as

$$
\varphi^{\prime}\left(x^{\prime v}\right)=\varphi\left(x^{v}\right)+\Delta \varphi\left(x^{v}\right)
$$

where $\Delta \varphi$ denotes the total variation for field

$$
\Delta \varphi=\delta \varphi+\left(\partial_{\alpha} \varphi\right) \delta x^{\alpha},
$$

we obtain the energy-momentum tensor [12]

$$
\begin{gathered}
T^{\mu}{ }_{v}=\frac{\partial L}{\partial\left(\partial_{\mu} \varphi\right)} \partial_{v} \varphi-2 \partial^{\mu}\left(\frac{\partial L}{\partial\left(\partial^{\alpha} \partial_{\alpha} \varphi\right)}\right) \partial_{v} \varphi \\
+\partial^{\mu} \partial_{\alpha}\left(\frac{\partial L}{\partial\left(\partial^{\alpha} \partial^{\beta} \partial_{\beta} \varphi\right)}\right) \partial_{v} \varphi-\delta^{\mu}{ }_{v} L .(10)
\end{gathered}
$$

Finally, the Hamiltonian density for the ansatz Lagrangian density can be written as [12]

$$
\begin{aligned}
T_{0}^{0}= & \frac{\partial L}{\partial\left(\partial_{0} \varphi\right)} \partial_{0} \varphi-2 \partial^{0}\left(\frac{\partial L}{\partial\left(\partial^{\alpha} \partial_{\alpha} \varphi\right)}\right) \partial_{0} \varphi \\
& +\partial^{0} \partial_{\alpha}\left(\frac{\partial L}{\partial\left(\partial^{\alpha} \partial^{\beta} \partial_{\beta} \varphi\right)}\right) \partial_{0} \varphi-L .
\end{aligned}
$$

Here we have derived the Hamiltonian density that will be used to reach our final purpose.

\section{Discussions}

Let us consider the one-dimensional Burgers equation. The general of the equation is usually written as

$$
\frac{\partial \Psi}{\partial t}=-\Psi \frac{\partial \Psi}{\partial x}+\lambda \frac{\partial^{2} \Psi}{\partial x_{2}},
$$

with $\lambda$ is interpreted as the constant of diffusion. By applying the Cole-Hopf transformation [4]

$$
\Psi(x, t)=-2 \lambda \frac{\partial}{\partial x} \ln u(x, t),
$$

and considering the traveling wave solution $\Psi(x, t)=\Psi(x-v t)$, leads to the analytical solution of the one-dimensional Burgers equation [This solution has been considered by Hans J. Wospakrik in the lecture but unpublished]

$$
\Psi(x, t)=\frac{2 v}{\left(\exp \left[\frac{v}{\lambda}\left(x-v t-x_{0}\right)\right]+1\right)} \text {. }
$$




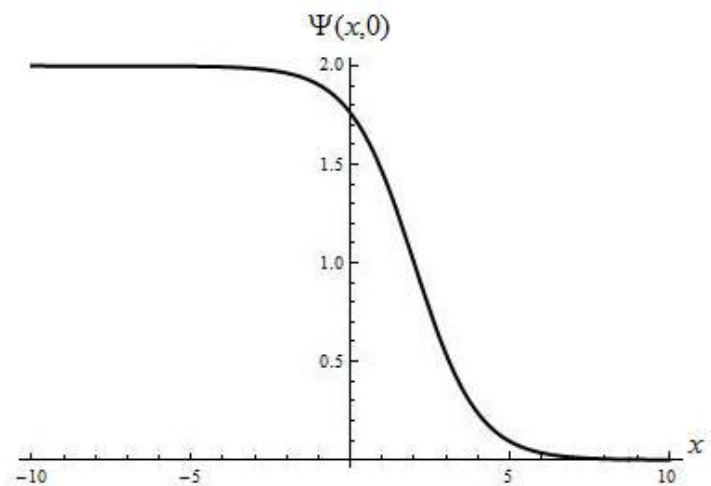

Figure 1. The static graphic of the Burgers equation for $v=\lambda=1$ and $x_{0}=2$.

Next, we are interested in considering the profile of the solution as follows

$$
\begin{array}{r}
\lim _{x \rightarrow \infty} \Psi(x, t)=0, \\
\lim _{x \rightarrow-\infty} \Psi(x, t)=2 v .
\end{array}
$$

From those two above characteristics, we directly conclude that the profile should be kink solution. Before we use Eq. (5) and (11), we firstly make transformation

$$
\Psi_{x}(x, t)=\varphi(x, t),
$$

where subscript denotes the partial derivative. With this transformation, the Burgers equation in Eq. (12) becomes

$$
\varphi_{x t}+\varphi_{x} \varphi_{x x}-\lambda \varphi_{x x x}=0 .
$$

Now, we introduce the ansatz Lagrangian density $L=L\left(\varphi, \varphi_{t}, \varphi_{x}, \varphi_{x t t}\right)$. By this choice, the Euler-Lagrange in Eq. (5) and Hamiltonian density in Eq. (11) will reduce

$$
\begin{gathered}
\frac{\partial L}{\partial \varphi}-\frac{\partial}{\partial t}\left(\frac{\partial L}{\partial \varphi_{t}}\right)-\frac{\partial}{\partial x}\left(\frac{\partial L}{\partial \varphi_{x}}\right)+\frac{\partial^{3}}{\partial x^{3}}\left(\frac{\partial L}{\partial \varphi_{x t t}}\right) \\
-\frac{\partial^{3}}{\partial t^{2} \partial x}\left(\frac{\partial L}{\partial \varphi_{x t t}}\right), \\
T_{0}^{0}=\frac{\partial L}{\partial\left(\varphi_{t}\right)} \varphi_{t}-\frac{\partial^{2}}{\partial x \partial t}\left(\frac{\partial L}{\partial\left(\varphi_{x t t}\right)}\right) \varphi_{t}-L .
\end{gathered}
$$

For seeking the explicit form both of the Euler-Lagrange equation and energymomentum tensor, we initially introduce the ansatz Lagrangian

$$
L=A \varphi_{x} \varphi_{t}+B \varphi_{x}{ }^{3}+C \varphi \varphi_{x t t},
$$

where $A, B$, and $C$ are the constants which have to be determined in order to satisfy the Burgers equation in Eq. (18). After substituting Eq. (21) into Eq. (19) and also considering Eq. (18), we find the explicit form of Lagrangian density as

$$
L=-\frac{1}{2} \varphi_{x} \varphi_{t}-\frac{1}{6} \varphi_{x}{ }^{3}-\lambda \varphi \varphi_{x t t} .
$$

Then, with the appropriate Lagrangian density written in Eq. (23), we easily obtain the explicit Hamiltonian density after substituting Eq. (22) into Eq. (20)

$$
T_{0}^{0}=\frac{1}{6} \varphi_{x}^{3}+\lambda \varphi \varphi_{x x t}+\lambda \varphi_{x t} .
$$

According to the Classical Field Theory (CST), the mass of the one-dimensional solitary wave solution can be formulated as [8]

$$
\mathrm{M}=\int_{-\infty}^{\infty} T_{0}^{0}(x, t),
$$

where for the $(1+1)$ dimensional space time the Hamiltonian density becomes

$$
T_{0}^{0}=\frac{1}{6} \varphi_{x}{ }^{3}+\lambda \varphi \varphi_{x t t}+\lambda \varphi_{x t} .
$$

By substituting the solitary wave solution in Eq. (14) to Eq. (11) by only considering (1+1) spacetime dimension, we find the resulted mass is divergent

\section{Conclusions}

We have calculated the mass of solitary wave solution of the one-dimensional Burgers equation depend on the formulation in the Classical Field Theory (CST) by integrating the Hamiltonian density over the space. In this case, the appropriate Hamiltonian density is derived by initially introducing the ansatz Lagrangian density that must satisfy the one-dimensional Burgers equation after substituting into EulerLagrange equation. To derive the Euler-Lagrange equation, we firstly minimize the action function which is the integral of the Lagrangian density over the space time.

We also have found that the result is divergent by using Mathematica. Since the result is divergent, we directly conclude that the Hamiltonian density does not describe the energy of system. In other words, the resulted mass has no physical meaning and can not be interpreted as the physical quantity.

\section{Acknowledgement}

We wish to thank all my colleagues that fully help us both for sending the international papers and exploring the discussions. 


\section{References}

[1] A. C. Scott, F. Y. F. Chu, and D. W. Mclaughlin, Proceedings of the IEEE, Vol. 61, No. 10, 1973.

[2] P. G. Drazin and R. S. Johnson, Solitons: an Introduction, New York: Cambridge University Press, 2002.

[3] J. M. Burgers, The Nonlinear Diffusion Equation, Boston: D. Rendel Publishing Company, 1974.

[4] N. A. Kudryashov and D. I. Sinelshchikov, arXiv: 0912.1542v1, 2009.

[5] Z. Feng, Phys. Lett. A 293 (2002) 57.

[6] J. L. V. Lewandowski, International Journal of Numerical Analysis and Modeling 3 (2006) 80.
[7] Ch. S. Rao and E. Saryanarayana, International Journal of Nonlinear Science 9 (2010) 290.

[8] H. J. Wospakrik, J. Theor. Comput. Stud. 4 (2005) 0308.

[9] A. Acus, arXiv : hep-ph/9901240v1, 1999.

[10]J. Schecter and H. Weigel, arXiv : hepph/9907554v1, 1999.

[11]M. Hadi and H. J. Wospakrik, arXiv : hepph/1007.0888v1, 2010.

[12]T. B. Prayitno, will be published in Jurnal Fisika, Universitas Negeri Semarang, 2012.

[13]L. H. Ryder, Quantum Field Theory, 2nd ed., Cambridge: Cambridge University Press, 1996. 